\title{
Changing Scenario in Management of Acute Coronary Syndromes in Females-Evidence from Recent Studies
}

\author{
Akshyaya Pradhan ${ }^{1}$ Pravesh Vishwakarma ${ }^{1} \quad$ Rishi Sethi $^{1}$
}

Address for correspondence Akshyaya Pradhan, DM, FAPSIC, FACC, FICA, Department of Cardiology, King George's Medical University, Lucknow 226003, Uttar Pradesh, India (e-mail: akshyaya33@gmail.com).

\author{
Abstract \\ Keywords \\ - Acute Coronary \\ Syndrome \\ - Cardiogenic shock \\ - Dual Antiplatelet \\ Therapy (DAPT) \\ - FFR (fractional flow \\ reserve) \\ - multivessel disease \\ - trials
}

Cardiovascular diseases are the leading cause of morbidity and mortality worldwide. Acute coronary syndrome (ACS) represents the most severe form of coronary artery disease (CAD) warranting urgent admission and intervention. Conventional teaching dictates that women are free from cardiovascular diseases in their reproductive age group. There is also a high prevalence of microvascular disease and atypical presentations in the fairer sex. Because of these factors, CAD is often under diagnosed and undertreated in women. They are also unfortunately underrepresented in major studies. But it is noteworthy that CAD remains the leading cause of death in both men and women alike. Evidence-based medicine and protocols have led to drastic improvement of outcomes of CAD and ACS. With close to one hundred trials presented at major cardiology meetings, it is impossible for the clinician to keep track of all of them. The authors describe briefly the recent studies in management of STEMI /ACS which have the potential to impact their daily practice. Hence, they also try to assess the impact of female gender on cardiovascular outcomes in these studies.

\section{Introduction}

Cardiovascular diseases (CVDs) are the leading cause of death and disability globally. Despite significant advances in therapy for coronary artery disease (CAD) over the past three decades, many gray areas exist. Because of their unique hormonal milieu, women are usually spared form CVD during their reproductive age group. There is also high prevalence of microvascular disease and atypical presentations in women. This had led to the perception that CAD is a disease of "men" leading to underdiagnosis and undertreatment in women. ${ }^{1}$ However, it is sad to note that CAD is the leading cause of death in men and women alike. The mortality following an acute coronary syndrome (ACS) is higher in women than in men. One of the putative reasons for this phenomenon can be that women are less aggressively treated with medical therapy such as $\beta$-blocker, aspirin, and reperfusion therapy. ${ }^{2}$

To add to our woes, women are often underrepresented in number in major studies. The outcomes of patients following ACS have improved with time. Various studies substantiating evidence-based medicine have been fueling these positive results. Each year a large number of trials make their way into the literature, and many of them are now being showcased at major meetings. We have selected primarily studies in STEMI and some in ACS, which have the potential to affect our routine practice. However, we have tried to discuss the results of female subgroups in all the studies that we will touch upon. However, because they were not prespecified subgroups prior to randomization in most, results need to be interpreted with caution. Guidelines and research studies are not "Gospel truth," and physicians need to have an individualized approach towards management of patients based on them.

\section{French Registry-ACS on the Rise in Women in Young}

Data from nationwide exhaustive French registry for admission in ACS were recently published. ${ }^{3}$ Over a period from 2004 to 2014, the age standardized admission for ACS in women aged $<65$ years increased by $6.3 \%$ from 28.1 to 29.9\%. Both ST-elevation myocardial infarction (STEMI) and non-ST-elevation myocardial infarction (NSTEMI) increased although the former increased more than latter. The trends in male population were not significant. The incidence for 
admissions for ACS beyond 65 years had significant decline paradoxically. The rise in ACS hospitalization in younger women is attributed to upward trends of smoking and obesity. This trend is alarming in the sense that female smoking rates have increased by two to five times in India., ${ }^{4,5}$

Data from ICMR-INDIAB-3 (Indian Council of Medical Research-India Diabetes 3) study has revealed that obesity (generalized and abdominal) is prevalent in India, with data ranging from 12 to $31 \%$. Interestingly, women had greater body mass index (BMI) than men in both urban and rural locations and female sex was an independent predictor of obesity. ${ }^{6}$

\section{CULPRIT-SHOCK-Keep It Simple}

CULPRIT-SHOCK (Culprit Lesion Only PCI versus Multivessel PCI in Cardiogenic Shock) trial compared a strategy of multivessel percutaneous coronary intervention ( $\mathrm{PCI}$ ) with an infract artery only PCI in 706 STEMI patients presenting with cardiogenic shock. ${ }^{7}$ The primary endpoint was a composite of death or renal failure leading to renal replacement therapy within 30 days after randomization. Patients randomized to infarct artery-only PCI group had $17 \%$ decrease in primary outcomes compared with the multivessel $\mathrm{PCI}$ group ( 45.9 vs. $55.4 \% ; p=0.01$ ). The risk of death (relative risk [RR] $0.84 ; p=0.03$ ) and renal-replacement therapy (RR 0.71; $p=0.07$ ) were also diminished in the culprit artery PCI group. Male-to-female ratio in the study was 3:1, and subgroup analysis did not reveal any difference in the outcomes based on gender. Reduction in mortality was the driving factor for benefits rather than low renal-replacement therapy. The absence of significant difference in renal-replacement therapy between groups seems intriguing despite the higher contrast medical use in multivessel arm.

It is a universal fact that cardiogenic shock in STEMI patients portends poor prognosis. Hence logically, guidelines and consensus statements up till now unanimously advocate for an approach of complete revascularization. This is primarily based on evidence from SHOCK (SHould we emergently revascularize Occluded Coronaries for Cardiogenic shock) trial done two decades ago, which may not reflect contemporary medical and interventional therapy. ${ }^{8}$ However, this study results do not favor such a strategy, and this needs to be accounted for by future guidelines. In absence of cardiogenic shock, however, the encouraging results from recent studies do seem to favor a complete revascularization approach. ${ }^{9}$

\section{Compare-Acute-Don't Shy Away from FFR in ACS}

The occurrence of multivessel disease in STEMI presents diagnostic and therapeutic challenges. Fractional flow reserve (FFR) has proven to be a quintessential tool in managing patients of stable angina with multivessel coronary disease. ${ }^{10} \mathrm{~A}$ few recent studies have showed that it is feasible to perform FFR in myocardial infarction (MI). ${ }^{11}$

Compare-Acute trial tested the role of FFR in patients with multivessel disease undergoing primary PCI for STEMI.
The study randomized 885 patients to receive either FFR guided complete revascularization or an infarct artery-only PCI. ${ }^{12}$ The female representation in both arms was 21 and $24 \%$, respectively. After 12 months, the primary endpoint (combination of death, nonfatal MI, revascularization, and cerebrovascular events) was significantly less in complete revascularization arm when compared with infarct artery PCI-only arm (7.3 vs. 20.5\%; hazard ratio [HR] $0.35 ; p<0.001$ ). On further analysis, it was seen that these impressive benefits were primarily driven by reduction in repeat revascularizations (5.1 vs. $16.6 \%$; HR $0.32 ; p<0.001$ ). The authors do not report any variation of results in various subgroups, and hence we believe the results are uniformly applicable to both sexes. The study stands in contrast to third Danish study, where FFR was performed a few days after primary PCI rather than during it. ${ }^{11}$ Nevertheless, the results suggest that FFR is feasible in acute STEMI patients and benefits primarily by reducing repeat revascularization.

\section{DAPT-STEMI and REDUCE-Keep It Short!}

Dual antiplatelet therapy (DAPT) for 1 year following PCI in STEMI patients is the standard of care. ${ }^{13}$ Such a combination has been shown to reduce stent thrombosis and reinfarctions. However, this strategy entails a high risk of bleeding events, and the question becomes more pertinent with advent of novel P2Y12 inhibitors. DAPT-STEMI and REDUCE studies tried to address the issue of bleeding with an abridged DAPT regimens.

DAPT-STEMI (Randomized, Open Label Trial of 6 Months Versus 12 Months DAPT After Drug-Eluting Stent in STEMI) trial tested a strategy of short (6 months) versus a standard (12 months DAPT) in 870 STEMI patients undergoing primary PCI. ${ }^{14}$ The primary endpoint (composite of death from any cause, MI, revascularization, stroke, and TIMI major bleeding) was not different in both arms at 18 months (HR $0.73 ; p=0.26$ ). Twenty-three percent of study population comprised of females. No sex-specific subgroup data analyses were reported, and hence study

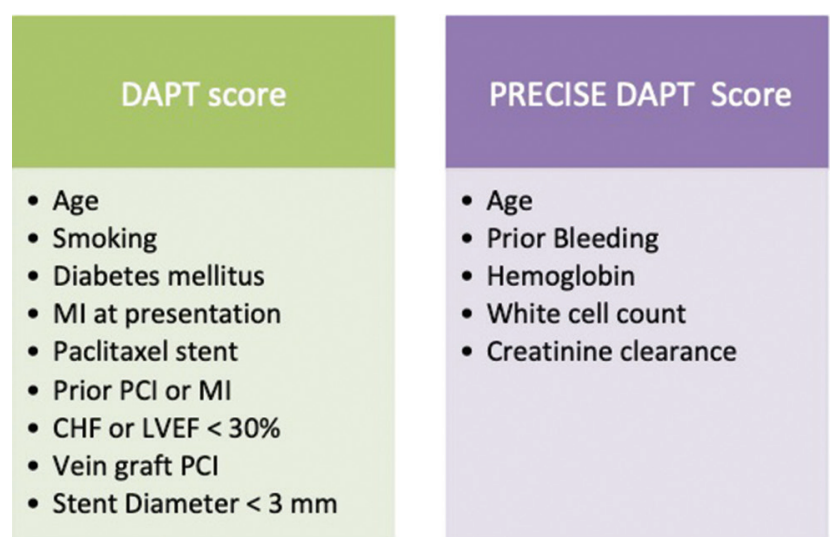

Fig. 1 Variables used in the PRECISE-DAPT and dual antiplatelet therapy (DAPT) score for continuing DAPT beyond 1 year of $\mathrm{PCl}$. A DAPT score $>2$ indicates favorable risk-benefit ratio with longer DAPT whereas a PRECISE DAPT score $<25$ corroborates with better bleeding risk profile with longer DAPT. CHF, congestive heart failure; LVEF, left ventricular ejection fraction; $\mathrm{MI}$, myocardial infarction; $\mathrm{PCl}$, percutaneous coronary intervention. 
conclusions apply to both sexes equally. It should be noted that the study underrepresented patients with diabetes and history of previous MI (14 and 5\%, respectively). Being strong predictors of stent thrombosis, both diabetes and history of previous MI have been incorporated in DAPT score ( - Fig. 1). DAPT score is a tool for predicting continuation of dual antiplatelet therapy after 1 year of PCI. ${ }^{13}$ Hence, the population in the study may have been at low risk of stent thrombosis, thereby leading to success of abridged antiplatelet regimen.

REDUCE (Randomized evaluation of short-term dual antiplatelet therapy in patients with acute coronary syndrome treated with the COMBO dual therapy stent) trial on similar note evaluated a short DAPT course (3 months) after PCI in patients with ACS vis-a-vis a standard DAPT course (12 months). ${ }^{15}$ Of the 1,496 study participants, almost one-half had STEMI. Patients underwent implantation of COMBO dual therapy stent (polymer drug-eluting stent [DES] loaded with sirolimus and endothelium promoting anti-CD34+ antibody) in contrast to zotarolimus-eluting stent used in DAPT-STEMI. Women comprised only $20 \%$ of study population. The primary endpoint examined was a composite of all-cause mortality, MI, stent thrombosis, stroke, target-vessel revascularization (TVR), and Bleeding Academic Research Consortium (BARC) bleeding $>1$. At the end of 12 months, event rates were similar in both the groups (short and longer DAPT course). Unlike the previous study, investigators reported results of subgroup analysis and all the spectrum of subgroups including age, sex, geographic region, and presence of diabetes had consistent results.

In both the studies, the short and ultra short DAPT regimens were noninferior to standard DAPT regimens regarding safety following DES implantation in an ACS setting.

\section{PRECISE-DAPT-Choose Wisely Beyond First Year}

Previously published studies on duration of DAPT following first year of PCI have demonstrated equivocal results. The DAPT score is a useful risk prediction tool to calculate the risk-benefit ratio of continuing DAPT beyond the standard 1 -year regimen. ${ }^{13}$

We have previously reviewed the derivation and predictive accuracy of PRECISE-DAPT (Predicting bleeding Complication In patients undergoing Stent implantation and subsEquent Dual Anti Platelet Therapy) score. ${ }^{16,17}$ In a nut shell, the score uses five variables (age, hemoglobin, white cell count, creatinine clearance, and history of prior bleeding) ( - Fig. 1 ). A score $<25$ predicts lower bleeding risk with preserved ischemic benefits. The 2017 ESC/EACTS (European Society of Cardiology/European Association for Cardio-Thoracic Surgery) Consensus document on DAPT also advises the use of PRECISE-DAPT score as a guide to DAPT durations following PCI. ${ }^{18}$ It is noteworthy that despite considered to be at high bleeding risk, female sex is not a part of either DAPT or PRECISE-DAPT score.

\section{Meta-analysis of Antiplatelet Function-Does Your Sex Matter?}

The impact of sex on platelet function has always been of subject of debate and controversy. The role of aspirin and clopidogrel in women has been previously questioned. ${ }^{19}$ With the advent of potent P2Y12 inhibitors, one could only speculate about sex-specific effects.

Interestingly, impact of sex on outcomes with newer antiplatelet therapies (P2Y12 inhibitors-ticagrelor and prasugrel) was studied by Lau et al in collaborative meta-analysis. ${ }^{20}$ They studied close to 87,000 patients from seven large and contemporary trials. Collectively these studies enrolled almost 24,500 women comprising about $27 \%$ of total population. Novel P2Y12 led to equal reduction in major adverse cardiovascular events (MACE) in both sexes ( 14 vs. $15 \%, p$ interaction $=0.95$ ). MI and stent thrombosis were also reduced in both sexes without any significant differences. Bleeding episodes were also increased in both sexes without any significant difference compared with standard therapy (HR 1.28 in women vs. 1.52 in men; $p$ interaction $=0.62$ ). This study failed to show heterogeneity of treatment effects based on sex.

\section{GEMINI-ACS-1-NOAC Are Safe}

The use of novel oral anticoagulants (NOAC) in an ACS setting has generally been disappointing with most trials facing a premature termination due to high rate of bleeding (REDEEM [Randomized Dabigatran Etexilate Dose-Finding Study in Patients with Acute Coronary Syndromes], APPRAISE [Apixaban for Prevention of Acute Ischemic Event], TRACER [Thrombin Receptor Antagonist for Clinical Event Reduction in Acute Coronary Syndrome], etc.). However, ATLAS ACS 2-TIMI 51 with rivaroxaban is an exception. This trial used low-dose rivaroxaban $(2.5$ and $5 \mathrm{mg}$ ) in patients with a recent ACS on top of DAPT and revealed ischemic benefits, but not without harm, that is, doubling of bleeding events. ${ }^{21}$

The GEMINI-ACS-1 (A Study to Compare the Safety of Rivaroxaban Versus Acetylsalicylic Acid in Addition to Either Clopidogrel or Ticagrelor Therapy in Participants With Acute Coronary Syndrome) trial randomized 3,037 patients of ACS (within 10 days of the event) to receive on top of a P2Y12 inhibitor either aspirin or low-dose rivaroxaban, an oral factor Xa inhibitor. ${ }^{22}$ The primary endpoint was thrombolysis in myocardial infarction (TIMI) clinically significant bleeding not related to coronary artery bypass grafting up to day 390. At 1 year follow-up, there was no difference of TIMI non-coronary artery bypass grafting (CABG) major bleeding between rivaroxaban arm and aspirin therapy (5.3 vs. $4.9 \% ; p=0.5840$ ). Although there was no signal of an increased risk of stent thrombosis in single antiplatelet group, the trial was not powered to assess differences in ischemic endpoints. Roughly, one-fourth of the trial population were females, and researchers did not find any difference of primary endpoint based on sex or age. Larger randomized and sufficiently powered studies will 
be required to establish the role of this novel "dual therapy" (NOAC plus single antiplatelet therapy) in ACS.

\section{VALIDATE-SWEDEHEART-Bivalirudin Has Run out of Steam.}

Although heparins are the choice of anticoagulant during $\mathrm{PCI}$, bivalirudin theoretically offers advantages in terms of less bleeding. However, after initial positive enthusiasm, the recent data from HEAT-PPCI (How Effective Are Antithrom botic Therapies in Primary Percutaneous Coronary Intervention) and MATRIX(Minimizing Adverse Haemorrhagic Events by Transradial Access Site and Systemic Implementation of AngioX) have raised questions over benefits of bivalirudin, especially in patients undergoing PCI by radial route and with use of potent P2Y12 inhibitors.

VALIDATE-SWEDEHEART (Bivalirudin versus Heparin in ST-Segment and Non-ST-Segment Elevation Myocardial Infarction in Patients on Modern Antiplatelet Therapy in the Swedish Web System for Enhancement and Development of Evidence-based Care in Heart Disease Evaluated according to Recommended Therapies Registry Trial) randomized 6,006 patients of ACS undergoing $\mathrm{PCI}$ with a potent P2Y12 inhibitor (ticagrelor or prasugrel) to heparin or bivalirudin. ${ }^{23}$ Almost $90 \% \mathrm{PCI}$ in the study were performed by radial route. The primary endpoint was death from any cause, MI, or major bleeding during 180 days follow-up. There was no difference in primary outcomes between bivalirudin arm and heparin arm at 30 days following PCI ( 8 vs. $7.2 \%, p=0.21$ ). Similar neutral trends were visible at 3 months, and the primary endpoints occurred with similar frequency between two groups ( 12.3 vs. $12.8 \%, p=0.54$ ). The results were consistent across both STEMI and non-STEMI (NSTEMI). Individual

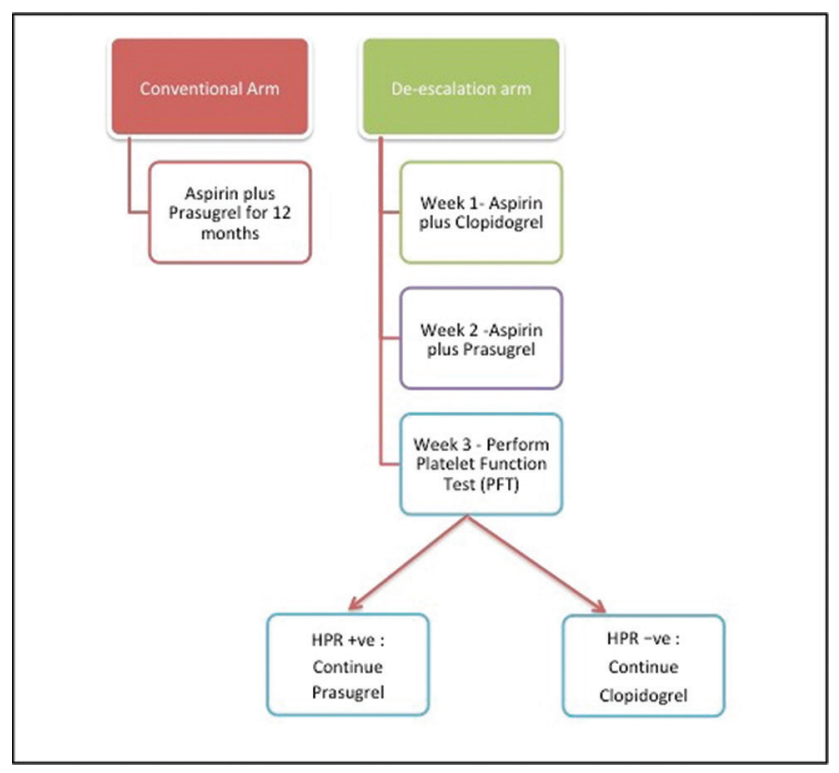

Fig. 2 De-escalation strategy used in TROPICAL-ACS study (HPRhigh on treatment platelet reactivity; based on multiplate analyzer testing; positive value $\geq 46 \mathrm{U}$ according to consensus definition). PFT, platelet function testing. endpoints of death, MI, major bleeding, and definite stent thrombosis were also not different between groups. Although the primary endpoint occurred less in female patients, the $p$ value for interaction between sex and drug intervention did not reach statistical significance. Despite the use of prolonged bivalirudin infusion in $65 \%$ patients, bivalirudin failed to show superiority to heparin. The recent ESC STEMI 2017 guidelines appropriately continue a class II recommendation to bivalirudin in STEMI. ${ }^{24}$

\section{TROPICAL-ACS-Time for Precision Medicine}

The enthusiasm for use of platelet function testing (PFT) to tailor antiplatelet therapy has been dampened by the lack of benefits from previous studies. ${ }^{25,26}$ TROPICAL-ACS study has thrown the spotlight back on PFT once again.

In the TROPICAL-ACS (Testing Responsiveness to Platelet Inhibition on Chronic Antiplatelet Treatment For Acute Coronary Syndromes Trial) study, a novel step-down regimen with aspirin plus prasugrel evaluated. ${ }^{27}$ The comparator arm was a standard uninterrupted regimen of the same drugs. The novel step-down regimen consisted of aspirin plus 1 week of prasugrel therapy, followed by 1 week of clopidogrel therapy. After 14 days, PFT was done, and those with high platelet reactivity (> 46 units) switched back to prasugrel whereas those without were maintained on clopidogrel (-Fig. 2). In the "tailored therapy" group, up to $40 \%$ showed insufficient platelet inhibition and had to be switched back to prasugrel. The primary endpoint was net clinical benefit (composite of cardiovascular death, MI, or BARC [Bleeding Academic Research Consortium] bleeding > grade 2) at 1 year. At study conclusion, event rates were $7 \%$ in guided step-down group versus $9 \%$ in standard group $(p=0.004$ for noninferiority, HR 0.81). Despite early de-escalation, there was no increase in ischemic events (cardiovascular [CV] death, MI, or stroke) in step-down group, but bleeding events were also not significantly decreased. Though there was male preponderance $(79 \%)$ in the sex distribution, the primary outcomes was no different when reassessed based on age and sex. Although cost intensive and time consuming, this trial results offer an alternative at least in patients deemed at high bleeding risk.

\section{SWEDEHEART Registry-Outcomes Are Improving in Women!}

Over the past two decades, significant advancements have occurred in technique and hardware for PCI. Simultaneously, pharmacotherapy of acute MI has evolved drastically as have protocols for management of MI. All these have translated into decline in deaths from STEMI as evidence from long-term data from nationwide SWEDEHEART (Swedish Web-system for Enhancement and Development of Evidence-based care in Heart disease Evaluated According to Recommended Therapies) registry. ${ }^{28}$ The 1 -year mortality from STEMI has declined from 22.1 to $14.1 \%$. 


\begin{tabular}{|c|}
\hline Key Messages \\
\hline Incidence of ACS is rising in females due to increased risk factors \\
\hline Comorbidities burden is higher in females with ACS. \\
\hline Use of antiplatelet provides equal protection without any signal of increased in females. \\
\hline FFR is useful in acute MI with multi vessel disease irrespective of gender. \\
\hline PRECISE DAPT score helps in deciding the duration of antiplatelet therapy in both sexes. \\
\hline $\begin{array}{l}\text { Oral anticoagulation can be safely added to antiplatelet regimen in ACS in both males \& } \\
\text { females. }\end{array}$ \\
\hline Shorter DAPT regimen following ACS is feasible and safe in both sexes. \\
\hline Bivalirudin doesn't better outcomes after PCl in either sex. \\
\hline Multivessel PCI has no vantage over culprit artery PCl in patients with cardiogenic shock. \\
\hline Outcomes form STEMI have improves in past decade and the effects are gender neutral. \\
\hline $\begin{array}{l}\text { Medical therapy is underutilized in females and their use leads to improves outcomes in } \\
\text { females }\end{array}$ \\
\hline $\begin{array}{l}\text { Females are often under represented in most studies and this disparity needs to be } \\
\text { addressed in future studies. }\end{array}$ \\
\hline
\end{tabular}

Fig. 3 Key messages and unmet needs. ACS, acute coronary syndrome; DAPT, dual antiplatelet therapy; FFR, fractional flow reserve; $\mathrm{MI}$, myocardial infarction; $\mathrm{PCl}$, percutaneous coronary intervention; STEMI, ST-elevation myocardial infarction.

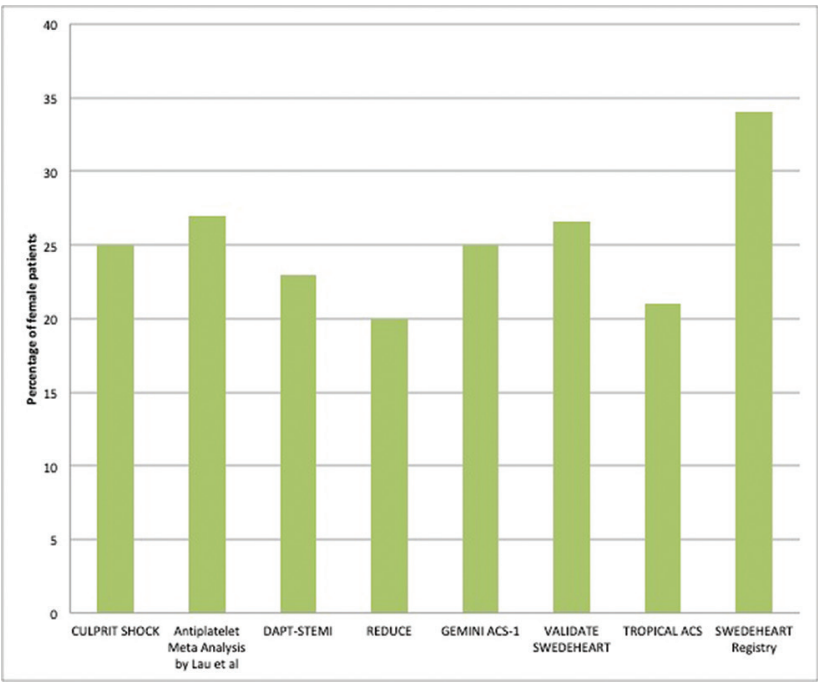

Fig. 4 Female representation in various studies in ACS. Except for the SWEDEHEART registry, in none of the randomized trials, the female proportion was $>30 \%$.

Impact of sex on outcomes of acute MI between 2003 and 2013 from the SWEDEHEART registry was published separately. ${ }^{29}$ Excess mortality at 6 months, 1 year, and 5 years was primary outcome measure analyzed. Women with acute MI (both STEMI and NSTEMI) were older and had more comorbidities such as diabetes, hypertension, etc. They had more unfavorable hemodynamics at presentation (lower systolic blood pressure [BP] and tachycardia) and were more likely to receive diuretics during stay. In this study, women with acute MI had no difference in all-cause mortality as compared with their male counterparts. There was a risk of excess mortality though, which was ameliorated by adherence to guideline directed medical therapy.

\section{Conclusion}

Management of ACS has evolved significantly over years and still undergoing changes as new data emerges. In 2017, many important studies focusing on various aspects of ACS made their way into the literature. The CULPRIT-SHOCK and Compare-Acute trials discussed management of multivessel disease in ACS. Tailoring antiplatelet therapy following ACS was the subject of discussion in DAPTSTEMI, REDUCE, TROPICAL-ACS, and PRECISE-DAPT studies. Lau et al in their meta-analyses demonstrated that novel antiplatelets are equally effective and safe in women. GEMINI-ACS and VALIDATE-SWEDEHEART concentrated on role of novel antithrombotic therapy following ACS. Long-term data of SWEDEHEART registry demonstrated improved outcomes in STEMI in both sexes over the past decades. Interestingly, a separate analysis of the registry revealed higher comorbidities in ACS women with excess mortality. However, judicious use of guideline-directed medical therapy was able to ameliorate the excess risk ( - Fig. $\mathbf{3}$ ).

Female participation in randomized controlled trials (RCTs) and registries is often inadequate ranging from 20 to $30 \%$ only (-Fig. 4 ). Subgroup analyses of most studies were available, and none of them showed any statistically significant difference in primary outcomes in this subset. This again reminds us of the fact that management of CAD leads to similar benefits in both sexes and that there is no reason to deprive women of therapy for ACS. CAD and ACS need aggressive and timely management irrespective of sex. We need to shed off the perception that it has no affliction for women. Underrepresentation in women and lack of large sex-specific studies is an unmet need that should be addressed in future research.

"The capacity to learn is a gift; the ability to learn is a skill; the willingness to learn is a choice."

-Brian Herbert

\section{Conflicts of Interest}

None.

\section{Source of Funding}

None.

\section{Acknowledgment of Grant Support}

None.

\section{References}

1 Sharma K, Gulati M. Coronary artery disease in women: a 2013 update. Glob Heart 2013;8(2):105-112

2 Jneid H, Fonarow GC, Cannon CP, et al; Get With the Guidelines Steering Committee and Investigators. Sex differences in medical care and early death after acute myocardial infarction. Circulation 2008;118(25):2803-2810 
3 Gabet A, Danchin N, Juillière Y, Olié V. Acute coronary syndrome in women: rising hospitalizations in middle-aged French women, 2004-14. Eur Heart J 2017;38(14):1060-1065

4 Goel S, Tripathy JP, Singh RJ, Lal P. Smoking trends among women in India: Analysis of nationally representative surveys (1993-2009). South Asian J Cancer 2014;3(4):200-202

5 Travasso C. Smoking prevalence among Indian women doubled in five years, study finds. BMJ 2014;349:g7512

6 Pradeepa R, Anjana RM, Joshi SR, et al; ICMR-INDIAB Collaborative Study Group. Prevalence of generalized \& abdominal obesity in urban \& rural India-the ICMR-INDIAB Study (Phase-I) [ICMR-NDIAB-3]. [ICMR-INDIAB-3] Indian J Med Res 2015;142(2):139-150

7 Thiele H, Akin I, Sandri M, et al; CULPRIT-SHOCK Investigators. $\mathrm{PCI}$ strategies in patients with acute myocardial infarction and cardiogenic shock. N Engl J Med 2017;377(25):2419-2432

8 Hochman JS, Sleeper LA, Webb JG, et al. Early revascularization in acute myocardial infarction complicated by cardiogenic shock. SHOCK Investigators. Should We Emergently Revascularize Occluded Coronaries for Cardiogenic Shock. N Engl J Med 1999;341(9):625-634

9 Elgendy IY, Mahmoud AN, Kumbhani DJ, Bhatt DL, Bavry AA. Complete or culprit-only revascularization for patients with multivessel coronary artery disease undergoing percutaneous coronary intervention: a pairwise and network meta-analysis of randomized trials. JACC Cardiovasc Interv 2017;10(4):315-324

10 Pijls NH, van Schaardenburgh P, Manoharan G, et al. Percutaneous coronary intervention of functionally nonsignificant stenosis: 5-year follow-up of the DEFER Study. J Am Coll Cardiol 2007;49(21):2105-2111

11 Engstrøm T, Kelbæk H, Helqvist S, et al; DANAMI-3-PRIMULTI Investigators. Complete revascularisation versus treatment of the culprit lesion only in patients with ST-segment elevation myocardial infarction and multivessel disease (DANAMI-3PRIMULTI): an open-label, randomised controlled trial. Lancet 2015;386(9994):665-671

12 Smits PC, Abdel-Wahab M, Neumann FJ, et al; Compare-Acute Investigators. Compare-Acute I. Fractional flow reserve-guided multivessel angioplasty in myocardial infarction. $\mathrm{N}$ Engl J Med 2017;376(13):1234-1244

13 O'Gara PT, Kushner FG, Ascheim DD, et al; American College of Emergency Physicians; Society for Cardiovascular Angiography and Interventions. 2013 ACCF/AHA guideline for the management of ST-elevation myocardial infarction: a report of the American College of Cardiology Foundation/American Heart Association Task Force on Practice Guidelines. J Am Coll Cardiol 2013;61(4):e78-e140

14 Kedhi E, Fabris E, Van der Ent M, et al. Six months versus 12 months dual antiplatelet therapy after drug-eluting stent implantation in ST-elevation myocardial infarction (DAPTSTEMI): randomised, multicentre, non-inferiority trial. BMJ 2018;363:K3793

15 Suryapranata H, De Luca G. REDUCE: A Randomized Trial of 3-Month vs 12-Month DAPT After Implantation of a Bioabsorbable Polymer-Based Metallic DES with a Luminal CD34+Antibody Coating in Patients with ACS. Late-breaking oral presentation at the 29th annual Transcatheter Cardiovascular Therapeutics scientific symposium, November 1, 2017

16 Costa F, van Klaveren D, James S, et al; PRECISE-DAPT Study Investigators. Derivation and validation of the predicting bleeding complications in patients undergoing stent implantation and subsequent dual antiplatelet therapy
(PRECISE-DAPT) score: a pooled analysis of individual-patient datasets from clinical trials. Lancet 2017;389(10073): 1025-1034

17 Pradhan A, Vishwakarma P, Sethi R. Landmark Trials in Cardiology in 2017-celebrating 40 years of angioplasty. Int J Angiol 2018;27(3):167-173

18 Valgimigli $M$, Bueno $H$, Byrne RA, et al; ESC Scientific Document Group; ESC Committee for Practice Guidelines (CPG); ESC National Cardiac Societies. 2017 ESC focused update on dual antiplatelet therapy in coronary artery disease developed in collaboration with EACTS: the task force for dual antiplatelet therapy in coronary artery disease of the European Society of Cardiology (ESC) and of the European Association for Cardio-Thoracic Surgery (EACTS). Eur Heart J 2018;39(3):213-260

19 Wang TY, Angiolillo DJ, Cushman M, et al. Platelet biology and response to antiplatelet therapy in women: implications for the development and use of antiplatelet pharmacotherapies for cardiovascular disease. J Am Coll Cardiol 2012;59(10):891-900

20 Lau ES, Braunwald E, Murphy SA, et al. Potent P2Y12 inhibitors in men versus women: a collaborative meta-analysis of randomized trials. J Am Coll Cardiol 2017;69(12):1549-1559

21 Mega JL, Braunwald E, Wiviott SD, et al; ATLAS ACS 2-TIMI 51 Investigators. Rivaroxaban in patients with a recent acute coronary syndrome. N Engl J Med 2012;366(1):9-19

22 Ohman EM, Roe MT, Steg PG, et al. Clinically significant bleeding with low-dose rivaroxaban versus aspirin, in addition to P2Y12 inhibition, in acute coronary syndromes (GEMINI-ACS-1): a double-blind, multicentre, randomised trial. Lancet 2017;389(10081):1799-1808

23 Erlinge D, Omerovic E, Fröbert O, et al. Bivalirudin versus heparin monotherapy in myocardial infarction. $\mathrm{N}$ Engl J Med 2017;377(12):1132-1142

24 Ibanez B, James S, Agewall S, et al; ESC Scientific Document Group. 2017 ESC Guidelines for the management of acute myocardial infarction in patients presenting with ST-segment elevation: the task force for the management of acute myocardial infarction in patients presenting with ST-segment elevation of the European Society of Cardiology (ESC). Eur Heart J 2018;39(2):119-177

25 Price MJ, Berger PB, Teirstein PS, et al; GRAVITAS Investigators. Standard- vs high-dose clopidogrel based on platelet function testing after percutaneous coronary intervention: the GRAVITAS randomized trial. JAMA 2011;305(11):1097-1105

26 Collet JP, Cuisset T, Rangé G, et al; ARCTIC Investigators. Bedside monitoring to adjust antiplatelet therapy for coronary stenting. N Engl J Med 2012;367(22):2100-2109

27 Sibbing D, Aradi D, Jacobshagen C, et al; TROPICAL-ACS Investigators. Guided de-escalation of antiplatelet treatment in patients with acute coronary syndrome undergoing percutaneous coronary intervention (TROPICAL-ACS): a randomised, open-label, multicentre trial. Lancet 2017;390(10104): 1747-1757

28 Szummer K, Wallentin L, Lindhagen L, et al. Improved outcomes in patients with ST-elevation myocardial infarction during the last 20 years are related to implementation of evidence-based treatments: experiences from the SWEDEHEART registry 1995-2014. Eur Heart J 2017;38(41):3056-3065

29 Alabas OA, Gale CP, Hall M, et al. Sex differences in treatments, relative survival, and excess mortality following acute myocardial infarction: National Cohort Study Using the SWEDEHEART registry. J Am Heart Assoc 2017;6(12):e007123 